No letter should be more than 400 words.

For letters on scientific subjects we normally reserve our correspondence columns for those relating to issues discussed recently (within six weeks) in the BMF.

We do not routinely acknowledge letters. Please send a stamped addressed envelope if you would like an acknowledgment.

- Because we receive many more letters than we can publish we may shorten those we do print, particularly when we receive several on the same subject.

\section{Breast screening in Britain and Sweden}

I have been employed in mammography and breast cancer screening since 1969, and, being the inventor of the single view mammography technique, I have some feelings about its use (15 October, p 932, 943, 969, 971). I have visited Britain many times, advising, teaching, reading papers, talking to people, and listening. This has certainly not made me an expert on Britain, but it has been impossible not to notice some important differences from Sweden. The reasons for these differences may cause serious problems if the British plan to emulate the Swedish screening model.

The incidence of breast cancer in Sweden has been stable during the past 10 years, with about 4200 new cases diagnosed each year. The mortality too is stable (though the age adjusted trend in mortality is downwards) with not quite 1500 patients dying each year. The mortality-incidence rate for $1982-4$ was $36 \%$ with screening involving only a small proportion of all women concerned. In 1960 the rate was nearly $50 \%$. I understand that the corresponding figure for Britain is $60 \%$ and that the average rate of advanced breast cancers is still astonishingly high -a figure of $35 \%$ in stage 3 was mentioned at a recent meeting in Oxford.

Swedes in general seem to have a solid confidence if not in the health care system as such at least in the ability of the medical profession to help them in case of serious disease. The tendency to delay consulting doctors has certainly decreased during recent decades. A sign of this is the decreasing Swedish mortality-incidence rate in breast cancer My interpretation of the high British mortalityincidence rate is that there might be a widespread scepticism regarding the ability of doctors to cure cancer at all-and this is certainly true so long as patients continue to appear with advanced disease.

A high compliance rate is extremely importan for the effectiveness of screening. Killer cancers are much more common among non-attenders. In Sweden, outside the big cities, $90 \%$ of women below 70 years of age participate when invited Furthermore, we are certain that this figure is correct because of our extremely efficient compute based population registries. No one can hide in Sweden today (this may be unfortunate in other respects, but not in the present one). Such registries do not exist in Britain. The nearest thing available is the general practitioner's card registries, which are notoriously unreliable, containing a considerable percentage of non-existent patients and not containing others who have no wish to be there, do not know that they should be, or hate things social in general.

There is also the general attitude towards the interests of society as opposed to those of the individual. It is probably not à la mode to talk about national characteristics but there are differences between Swedes and Britons in these attitudes. In general Swedes are rather docile and do not tend towards civil disobedience. Ninety per cent of the Swedish population vote on election days. In Britain there is a different tradition with a strong emphasis on the importance of the rights of the individual and a deep rooted suspicion of and resistance to ordinances from above. What percentage of the British population vote? Fifty five or sixty per cent? (How is it possible to know without registries?) The social structures of Sweden and Britain are also different. The Swedish taxation system is aimed at minimising class differences and has been partially successful at least in reducing obvious poverty to a low level. Such class differences are much more in evidence in Britain. The poverty related part of the problem-ignorance, alienation, suspicion - combined with the slightly paranoic civic attitude may well be responsible for a largely ill founded lack of confidence in medicine and thus the high rate of advanced cancers, the strikingly high breast cancer death rate, the lack of efficient population registries, the large holes in general practitioners' registries, and the low compliance in the pilot screenings. Whether these attitudes can be changed over a generation is questionable. But can some confidence be established as a partial goal?

Under such circumstances I doubt that breast screening in Britain can be really effective until a very long time has passed except as a means of increasing the awareness of women of the potential benefit of early self diagnosis and of increasing their confidence in the ability of medicine to cure localised breast cancer. Rather than beginning with the screening project, perhaps a very ambitious and loud sustained campaign directed at the less fortunate and aimed at bridging the confidence gap should be considered as a first step to decrease Britain's depressingly high mortality from breast cancer. Such a campaign is certainly necessary to make women appear at the screening centres, which in turn is necessary for effective screening.

BENGT LUNDGREN

Mammography Department, Gävle County Hospital, 80189 Gävle, Sweden

\section{Controversy over mammographic screening}

My reading of the scientific evidence from controlled studies of mammographic screening in other countries suggests that for women aged 55 and above screening seems to reduce mortality from breast cancer; that we do not yet know whether one or two view mammograms are necessary; that we do not know the most appropriate screening interval; and that the best way to treat lesions detected on screening is still unknown. Furthermore, both the number of lives saved and the cost and benefit for those lives saved still seem to be uncertain (10 September, p 650).

May I suggest that these questions are all somewhat academic unless one other fundamental problem is tackled - that of compliance and regular attendance. As acceptance of an invitation to screening is a problem among the more health conscious older Swedish women in the Malmö study (15 October, p 943) it is likely to be a vast problem in Britain. Examination of the sociodemographic characteristics of attenders and nonattenders at breast screening clinics in Britain is not especially encouraging. One recent study of attendance at a regional screening clinic found that only $18 \%$ of the sample were over the age of 55 years - that is, women most at risk did not attend. Another study looking at psychological morbidity in women participating in the Edinburgh breast screening trial reported that only $67 \%$ women accepted the invitation to screening. ${ }^{2}$

It is important to consider the psychological factors or health beliefs affecting the acceptance of any health promotion or illness prevention behaviour. One model suggests that women will attend for screening only if they believe $(a)$ in personal susceptibility to breast cancer; $(b)$ that the disease would have at least a moderately severe impact on some component of life; $(c)$ that breast screening would reduce either susceptibility to the condition or its severity and the necessity for aggressive treatment; $(d)$ that the disease could be present even if they themselves are not experiencing any symptoms; and $(e)$ that treatments are effective. $^{3}$

Precisely how to educate a wide cross section of women from different backgrounds, all of whom might also have differing health beliefs, presents health educationalists and others with a formidable challenge. We need much more basic research into, for example, the appropriate tone or anxiety provoking "pitch" of the letters of invitation. We also need to think of appropriate ways of motivating women to continue to come for screening and to practise breast self examination between screening invitations. Other important factors that migh influence uptake are the person who sends out the invitation and the site chosen for screening.

The huge investment of both money and manpower into the screening programme demands that proper attention should also be paid to the psychosocial factors that are bound to affect its success or otherwise, and I hope that research money will be available for these important issues to be examined adequately.

LESLEY FALLOWFIELD

Department of Psychiatry

The London Hospital Medical College, London E1 2AD 
1 Owens RG, Daly J, Heron K. Psychological and social characteristics of attenders for breast screening. Psychology and Healt 1987;1:303-13.

2 Dean C, Roberts M, French K, Robinson S. Psychiatric morbidity after screening for breast cancer. I Epidemiol Community Health 1986;40:71-5.

3 Rosenstock IM. Why people use health services. Milbank Memorial Fund Quarterly 1966;44:94-127.

\section{Malaria prophylaxis and epilepsy}

Drs D R Fish and M L E Espir reported on tonicclonic seizures (27 August, p 526) in four women taking chloroquine for malaria prophylaxis either alone or in combination. They concluded that a specific inquiry should be made for a history of epilepsy when malaria prophylaxis is being considered and that patients with epilepsy should be advised about the risk of antimalarial drugs provoking seizures.

The cases reported are briefly described. In three of them the time between the last dose and the seizures is not stated. We believe that many epileptic patients are seen annually at the Institute of Neurology, where the authors work. It would hardly be surprising to find a small percentage among these who take antimalarial drugs as well The time span during which the four patients were seen and the annual number of epileptic patients at the institute are not stated. Therefore the likelihood of a causal relation between the seizures and the antimalarial prophylaxis cannot be estimated.

In Sweden, as in the United Kingdom, there have not been any reports to the Adverse Drug Reaction Committee about convulsions during chloroquine treatment or prophylaxis. It is very difficult to estimate how many Swedish travellers have used chloroquine for malaria prophylaxis. I is estimated from official sales statistics (Nationa Corporation of Swedish Pharmacies, 1987) that 12000 rheumatic patients undergo maintenance treatment with chloroquine each year, many of whom have certainly had a history of seizures. Furthermore, the dose given and the chloroquine concentrations are much higher in these patients than in travellers undergoing malaria prophylaxis Considering the nature of epilepsy with spontaneous recurrence and the lack of reported adverse reactions we do not regard it necessary to ask travellers receiving chloroquine alone about possible previous seizures.

It may be noteworthy that chloroquine was combined with pyrimethamine and a sulphonamide in three of the four cases reported and that one of these had no evidence of a low seizure threshold. This might indicate a risk for convulsions when these drugs are taken in combination. Nevertheless, the number of cases is small and the pattern of prescription of malaria prophylaxis in the catchment area is not stated in the report. Rather than recommending restrictions in malaria prophylaxis or travelling for people with epilepsy, the authors' report should alert prescribers to look for and report possible further cases of seizures in connection with malaria prophylaxis.

URBAN HELLGREN LARS ROMBO

Department of Infectious Diseases,

Karolinska Institutet,

11489 Stockholm,

Sweden

AUTHORS' REPLY,-The seizures in the four women reported on occurred during once a week antimalarial prophylaxis and were seen by us within a 15 month period. With regard to the intervals between the seizures and the last dose of the drugs shown in our original table, these were as follows: case 1, 5 days; cases 2 and 3, 1 day; and case 4 , two tonic-clonic seizures during the week after the second dose of chloroquine and fifth dose of Fansidar. Although in case 1 the subsequent electroencephalogram showed generalised $3 \mathrm{~Hz}$ spike and wave activity, only the second and third patients had a history of epilepsy.

Like other neurologists, we see numerous patients with epilepsy, some new referrals, others for review. The prevalence figures in the United Kingdom vary, depending on definitions, from 0.5 to $2.0 \%$, but we do not know how many patients have taken antimalarial drugs prophylactically or how many also have rheumatoid arthritis treated with chloroquine. We suspect that these numbers are small and that an increase in fit frequency may not be recognised as an adverse drug effect. We think that the association between malaria prophylaxis and the tonic-clonic seizures in our four patients is unlikely to have been due to chance for the reasons given in our report. Our third patient took chloroquine alone, but combinations of drugs might carry a higher risk, although this is speculative in view of the small numbers reported so far

We did not conclude that restrictions should be placed on patients with epilepsy. We suggested that an adequate history should be taken before prescription and that patients with epilepsy should be warned that there may be a risk of seizure provocation. The action taken would depend on the destination, reasons for travel, and the implications for the patient if a seizure occurred. We agree it would be helpful if the statistical risk could be determined and a population based study would be interesting. This may not, however, be the best way of detecting an adverse drug effect if this occurs in only a small proportion. Our paper was intended to bring the cases to the notice of prescribers and alert them to the possible danger for patients known to have epilepsy. We agree that further cases of seizures in connection with malaria prophylaxis should be reported.

Since our paper was published we have been informed about two further examples, both young men with no previous or family history of epilepsy. Firstly, a 17 year old man (reported by Professor W I McDonald) had a tonic-clonic seizure on the first day of his holiday in Egypt this August, 24-36 hours after taking a second weekly dose of chloroquine (two tablets) and Maloprim (1 tablet) His subsequent electroencephalogram was normal. Secondly, a 19 year old seaman on service (reported by $\mathrm{Dr} T \mathrm{D}$ L Thomas) lost vision, developed urticaria, and then had a tonic-clonic seizure 10 minutes after taking a first dose of chloroquine alone in July. He was said to have an inherited food allergy.

D R FISH M L E ESPIR

Institute of Neurology, Queen Square,

London WCIN 3BG

\section{Chlamydia: One step forward or two backwards?}

Dr Ian Alexander (24 September, p 791) highlighted the need for both diagnostic facilities and contact tracing in specialised departments and primary health care teams if chlamydial infections are to be correctly managed

The general practice team at Llanederyn Health Centre has been using the fluorescein labelled monoclonal antibody test MicroTrak (Syva) for nearly two years. ${ }^{\prime}$ Its use in general practice has been documented. ${ }^{2}$ The diagnosis of two cases of neonatal chlamydial conjunctivitis within a short time led us to look more closely at the problem of chlamydia in the practice with this new technique.

During the past 12 months we investigated 260 women presenting at the health centre with vaginal symptoms, of whom $15(6 \%)$ had positive results with MicroTrak tests. The practice list size is about 7000 in a mixed council and private suburban housing estate, with the population being predominantly white of social class III manual and below.

Though our detection rate is $6 \%$ for chlamydia in women with symptoms and not as high as that in genitourinary medicine clinics, in view of the potentially serious sequelae of chlamydial infections we consider it important that primary health care teams have access to diagnostic facilities such as MicroTrak.

We agree with Dr Alexander that contact tracing is not easy, but we think that it is in danger of being overcomplicated and general practitioners may be given the impression that contact tracing is something they should not be doing. At the heart of all contact tracing is the provision of information to patients about their infection. For chlamydia they will need to know about its potential long term complications, that it may be asymptomatic, and that their current and recent sexual partners may well be infected without knowing it and therefore still be a source of infection. The necessity of investigating their partners will be discussed, either by referral to the local genitourinary medicine clinic or by the general practitioner. Ultimately it is the patients' responsibility, once they have all the necessary information, to ensure that contacts are traced and the chain of infection broken.

If contact tracing has not been successful the general practitioner can discuss this with patients when they reattend the surgery for other problems: continuous care of patients is one advantage of contact tracing by family doctors.

If sexually transmitted diseases, particularly chlamydia, are to be dealt with more successfully than they are at present genitourinary medicine specialists will need to continue the educational service they provide to primary health care teams. Only with improved cooperation between primary and secondary health care services and patients may we hope to bring one of the major health problems of the 1980s under control.

PENNY OWEN

Department of General Practice,

University of Wales College of Medicine

Llanederyn Health Centre,

Cardiff CF3 7PN

Public Health Laboratory Service,

JULIA MUNRO

Cardiff

Department of Epidemiology and

Community Medicine,

Cardiff

1 Thomas BJ, Evans RT, Hawkins DA, Taylor-Robinson D. Sensitivity of detecting Chlamydia trachomatis elementary bodies in smears by use of a fluorescein-labelled monoclonal antibody: comparison with conventional chlamydial isolation. f Clin Pathol 1984;37:812-6.

2 Longhurst HJ, Flower N, Thomas BJ, et al. A simple method for the detection of Chlamydia trachomatis infections in general practice. I $R$ Coll Gen Pract 1987;37:255-6.

\section{Fertility and legal abortions as performance indicators}

Professor Michael Clarke (1 October, p 832) suggests the use of total period fertility and abortion rates as performance indicators. The weakness of this follows from the assumptions about the meaning of different rates to different cohorts of women at different stages in their lives, and I suggest that it makes unnecessarily complicated what are basically simple issues.

We know from various studies of abortion and family planning that the group at greatest risk of services not meeting their needs is the 15-19 age group ${ }^{1-3}$ and that from a numerical point of view the 20-24 age group accounts for the biggest number of abortions, reflecting the experience of a population that is very sexually active but not yet 